\title{
Functionalization of carbon nanotubes and mechanical characterisation of bio-based epoxy nano-composites
}

\author{
A. Singh*, C. Sguazzo*, C.F.R.A.C. Lima ${ }^{\dagger}$, L.M.N.B.F. Santos ${ }^{\dagger}$, P.J.S. Tavares* and \\ P.M.G.P. Moreira* \\ *Laboratory of Optics and Experimental Mechanics (LOME), \\ Institute of Science and Innovation in Mechanical and Industrial Engineering (INEGI) \\ Rua Dr. Roberto Frias, Campus da FEUP, 400 4200-465, Porto, Portugal \\ email: csguazzo@inegi.up.pt, webpage: http://www.inegi.up.pt \\ ${ }^{\dagger}$ Department of Chemistry and Biochemistry, \\ Faculty of Science of University of Porto, \\ R. Campo Alegre, 687, 4169-007 Porto, Portugal
}

Keywords: Carbon nanotubes, Composites, Functionalization, Mechanical Characterization, Polymers.

\begin{abstract}
Chemical functionalization of multiwalled carbon nanotubes (MWCNTs) is important from the view point of polymer based composite manufacturing. As pristine MWCNTs has certain disadvantage such as they have lower dispersion, they are hydrophobic and are not readily soluble in a solvent, these characteristics makes them unreliable candidate for most of the industrial applications. By doing chemical functionalization of MWCNTs, these shortcomings can be overcome, and the MWCNTs can be used as a filler in composite manufacturing. This has the advantage of better nanofillers' dispersion and provide the better interfacial bonding.

In this study, MWCNTs are functionalized by the carboxylic group, chemical functionalization of MWCNTs is an optimization problem, governed by parameters like mixture acid concentration, temperature, time of heating and amount of MWCNTs used. Material characterization of MWCNTs is done and test specimens are manufactured according to different concentrations of MWCNTs within a bio-based epoxy resin. Mechanical properties are then compared according to different concentrations. These mechanical and material characterizations increase the understanding of chemical functionalization by carboxylic group and the influence of the concentration of MWCNTs dispersed within the bio-based resin matrix.
\end{abstract}

\section{INTRODUCTION}

Use of composite materials in aircraft industry comes with certain advantages such as light weight, high temperature resistant, high strength to weight ratio, resistant to corrosion, flexibility, in association with their design. Furthermore, the use of bio-based resins is on the rise to reduce the carbon footprints in different engineering fields [1], including the aeronautic sector. Recently, research is focused on the use of carbon nanotubes to be incorporated into the polymer matrix as a filler material to increase multiple physical properties. A significant boost in the carbon nanotubes research field [2] came after an impactful research paper by Sumio and Iijima in 1991 [3]. Carbon nanotubes are very promising candidates because of properties such as high strength, low density, high conductivity, chemical inertness and low-dimensionality, thus their use is open to diverse field applications [4]. Carbon nanotubes in its pristine form are difficult to disperse within the polymer matrix due to the presence of strong van der Waals forces, which keep them as an agglomerate [5]. To improve their dispersion capability within polymer matrices, chemical functionalization can be 
applied. Modification techniques may be broadly categorized as covalent and non-covalent functionalization [6].

In this work, covalent functionalization was performed by using sulphuric and nitric acid (mixture acid), resulting in the functionalization of the surface of the MWCNTs by the carboxylic group, in order to improve the dispersion of pristine MWCNTs within an epoxy matrix. Indeed, the compatibility of carboxylic group with the epoxy molecules helps to increase the dispersion and the interfacial bonding with the epoxy matrix. Particularly, within the chemical functionalization by mixture acid, sulphuric acid creates a defect site at the surface of the MWCNTs and nitric acid oxidized these defects [7], thus, grafting the carboxylic group on the walls of carbon nanotubes. Furthermore, this changes the $\mathrm{sp} 2$ hybridization of multi-walled carbon nanotubes into $\mathrm{sp} 3$ hybridization and makes them more stable.

Different key points characterize the chemical functionalization such as: higher curvature MWCNTs are more reactive at the site, smaller diameter MWCNTs are easier to functionalize, ends of the MWCNTs are more reactive than the side walls, MWCNTs with defects are more susceptible to functionalization $[4,7]$. Furthermore, there are certain parameters that control the functionalization process which are the concentration of the acids used, the temperature of the solution, the duration of the acid treatment and the ratio of MWCNTs to acid.

In this work, bio-sourced epoxy resin was used as matrix for the nanocomposites to produce. Material characterization of the pristine and functionalized MWCNTs was carried out and the process of fabrication of the nanocomposite was optimized. Finally, tensile mechanical properties were analysed according to different concentrations of both pristine and functionalized MWCNTs.
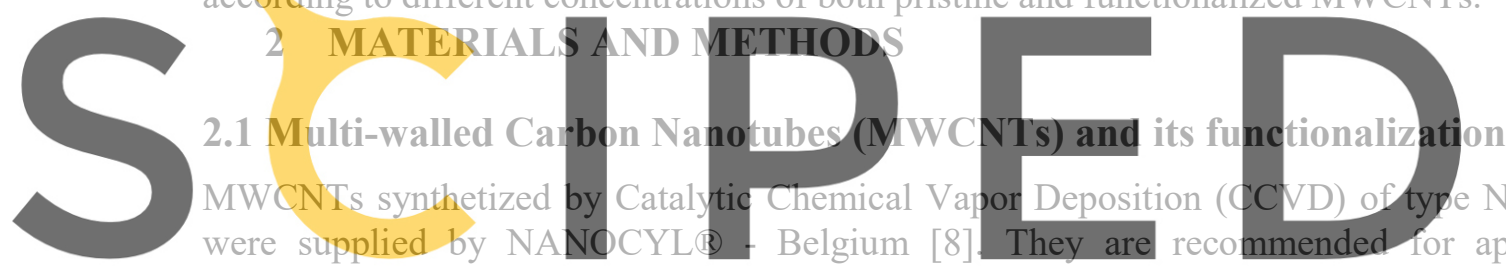

were supplied by NANOCYL(1) - Belgium [8]
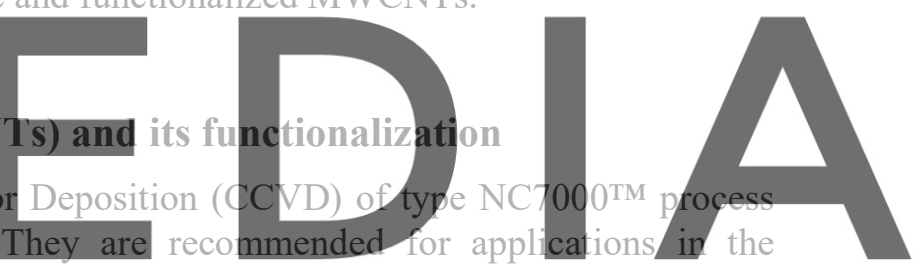

transportation including the aeronautic sector and for EMI-shielding, because of their high electrical

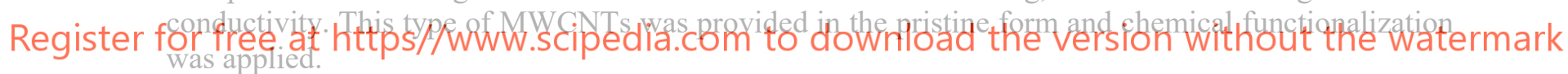

Pristine MWCNTs were functionalized in a mixture acid of 3 part of concentrated sulphuric acid and 1 part of concentrated nitric acid. A quantity of $100 \mathrm{mi}$ of mixture acid was heated to a temperature of $90{ }^{\circ} \mathrm{C}$ and then maintained for a certain time. The pristine MWCNTs in a quantity of $1 \mathrm{~g}$ was added to the mixture acid and five different synthesis were carried out using time of heating: 15, 30, 45, 60 and 90 minutes in a reflux $[9,10]$. After the synthesis, the sample was diluted in a deionized water (1L) and then filtered in a vacuum by means of a $0.22 \mu \mathrm{m}$ membrane filter. The resultant solid was then washed with deionized water until the $\mathrm{pH}$ reached neutral value. Finally, MWCNTs were dried in oven at 60 ${ }^{\circ} \mathrm{C}$ for $24 \mathrm{~h}$ and weighed afterwards. The dried MWCNTs were added to dimethylformamide (DMF) and the soluble part was then separated from the insoluble part.

\subsection{Thermogravimetric Analysis (TGA)}

The thermal behaviour of both pristine and functionalized MWCNTs was studied by means of TGA analysis. The samples of MWCNTs were heated from the room temperature to $900{ }^{\circ} \mathrm{C}$ in a nitrogen atmosphere with a gas flow rate of $200 \mathrm{ml} / \mathrm{min}$ characterised by a heating rate of $10^{\circ} \mathrm{C} / \mathrm{min}$. Then an isotherm condition was kept at $900{ }^{\circ} \mathrm{C}$ for 10 minutes and followed by cooling down to room temperature at a heating rate of $10^{\circ} \mathrm{C} / \mathrm{min}$. 


\subsection{Scanning Electron Microscopy (SEM)}

Both pristine and functionalized MWCNTs were prepared for the SEM analyses, which were performed at were performed at CEMUP (Centro de Materiais da Universidade do Porto), Porto, Portugal. To visualize the MWCNTs in the SEM, thin film of MWCNTs was deposited over the Indium Tin Oxide (ITO) coated glass (to make them conductive). The pristine MWCNTs and the ones functionalized in 45 minutes and 90 minutes were analysed at a scale of $2 \mu \mathrm{m}$.

\subsection{Preparation of MWCNTs based epoxy nanocomposite}

In this study the epoxy/hardner resin system used for producing the nanocomposites has the commercial name of SR GreenPoxy® 33/SZ 8525 and was supplied by Sicomin - France [11]. SR GreenPoxy ${ }^{\circledR} 33$ has a bio-based carbon content with $35 \%$ of its molecular structure coming from plant origin. According to the TDS, viscosity of the resin at $20^{\circ} \mathrm{C}$ and $25^{\circ} \mathrm{C}$ is $3240 \mathrm{mPa} \cdot \mathrm{s}$ and $1780 \mathrm{mPa} \cdot \mathrm{s}$ respectively. Viscosity of the hardener at $20^{\circ} \mathrm{C}$ and $30^{\circ} \mathrm{C}$ is $33 \mathrm{mPa} \cdot \mathrm{s}$ and $25 \mathrm{mPa} \cdot \mathrm{s}$ respectively. In the following the description of the method developed for fabricating the resin-based nanocomposites id reported. MWCNTs were added to the acetone and sonicated for 30 minutes, for making them easy to disperse and for reducing the viscosity of the mixture with the epoxy resin. Epoxy was added to this mixture and sonication was carried out for another 30 minutes. After that, the mixture was subjected to magnetic stirring for $3 \mathrm{~h}$ at $80{ }^{\circ} \mathrm{C}$ in order to completely remove the acetone from this mixture. Degassing of the mixture was done for 15 minutes to ensure the removal of any air bubbles trapped because of previous processes. At this step, hardener was added to the mixture and was magnetic stirred for 15 minutes without heating. Sonication was performed for another 2 minutes and then degassing was done for

$120^{\circ} \mathrm{C}$ for 45 minutes. temperature.
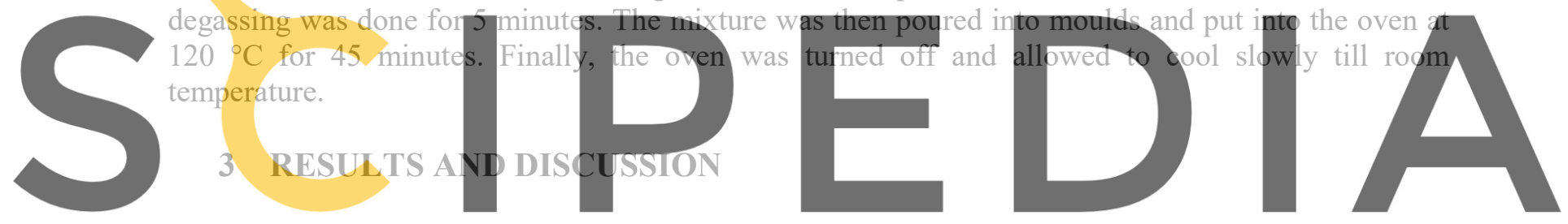

3.1 Yield Time vs the Time of Synthesis

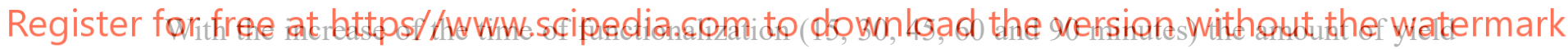
of MWCNTs which was acquired after synthesis followed an exponential decay, which is in accordance with the first order reaction kinetics. This is shown in Figure 1. Exponential decay followed the Equation 1 below, where $\mathrm{x}$ is the time of synthesis and $\mathrm{y}$ is the amount of yield after the functionalization.

$$
y=973.21 e^{-0.015 x}
$$

\subsection{Hydrophilic behaviour of MWCNTs after functionalization}

Hydrophilicity of MWCNTs was confirmed by mixing the pristine and functionalized MWCNTs into two separate bottles containing water and dichloromethane (DCM). Pristine MWCNTs showed to have an affinity towards DCM while MWCNTs showed to have a hydrophilic nature and got attracted towards water at the top. Thus, confirming the change of behaviour from hydrophobic to hydrophilic. This change of behaviour was also used to separate the non-functionalized part from the functionalized one. The functionalized product was dissolved in the DMF and then functionalized and nonfunctionalized parts were separated. Separated functionalized product gave the same percentage of functionalized product after each synthesis, which followed the previously reported exponential decay. This led to a conclusion that with the increase of time of synthesis, the overall yield of MWCNTs decreased but the percentage of functionalized product inside the yield quantity remained the same. It 
was observed that the time of reaction consumed more pristine MWCNTs but did not change the final quantity of functionalized MWCNTs. Therefore, the first reproducible functionalization time of 30 minutes was chosen for producing the bulk functionalized MWCNTs. The time of 15 minutes of synthesis was not used as it was not as reproducible as the 30-minute functionalization reaction.

Table 1: Percentage of functionalized MWCNTs soluble in DMF

\begin{tabular}{cc}
\hline Functionalization Time (minute) & Percentage of pure functionalized MWCNTs \\
\hline $\mathbf{1 5}$ & $13 \%$ \\
\hline $\mathbf{3 0}$ & $14 \%$ \\
\hline $\mathbf{4 5}$ & $14 \%$ \\
\hline $\mathbf{6 0}$ & $10 \%$ \\
\hline $\mathbf{9 0}$ & $15 \%$
\end{tabular}

\subsection{TGA results of pristine and functionalized MWCNTs}

TGA results showed the degree of functionalization of MWCNTs with the carboxylic group and the results were compared with the pristine MWCNTs. Pristine MWCNTs showed a decrease of the weight to $13.74 \%$ whereas the functionalized MWCNTs weight decreased to $25.41 \%$. This increase in weight loss from pristine to functionalized MWCNTs could be due to the presence of carboxylic group at the surface of MWCNTs. Figure 2 shows the mass loss for different samples.
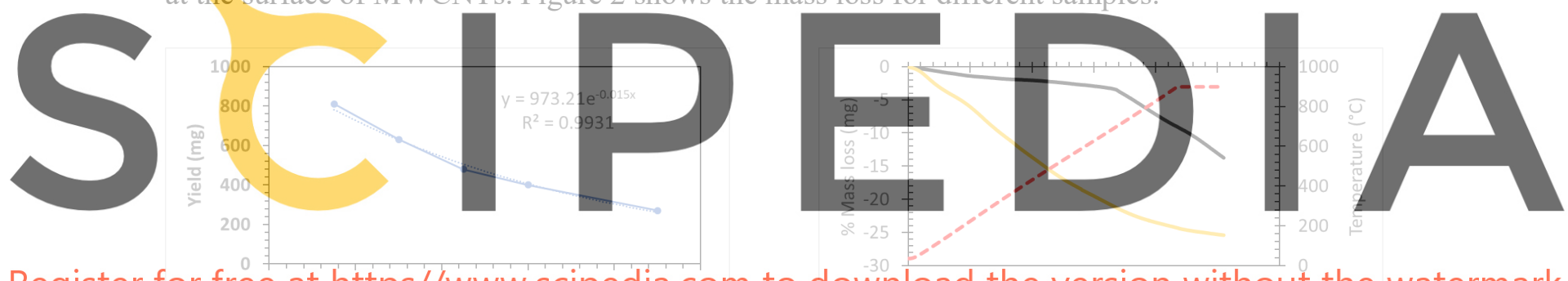

Register for free at https/fawwwoscipedia.com to downbad the version withodt the watermark Time (min)

Figure 1: Amount of yield vs time of functionalization

Figure 2: TGA results of pristine and functionalized MWCNTs

\subsection{SEM analysis}

The results of SEM analyses are reported in this paragraph. Figure 3a) shows the pristine MWCNTs at $2 \mu \mathrm{m}$ length scale: they appeared in the form of agglomerates, long seamless wires and appeared entangled together due to the weak van der Waals forces. Figure $3 \mathrm{~b}$ ) and $3 \mathrm{c}$ ) shows SEM images of the 45-minute functionalized MWCNTs and 90-minute functionalized MWCNTs. From the figure, the MWCNTs appeared well dispersed and the length of the MWCNTs was shorter than the pristine ones. It was observed that the length of MWCNTs decreased after the acid treatment, but they were not anymore in the form of agglomerates, thus resulting well dispersed. Also, by increasing the time of functionalization from 45 minute to 90 minutes, the quantity of functionalized MWCNTs appeared to be reduced. 

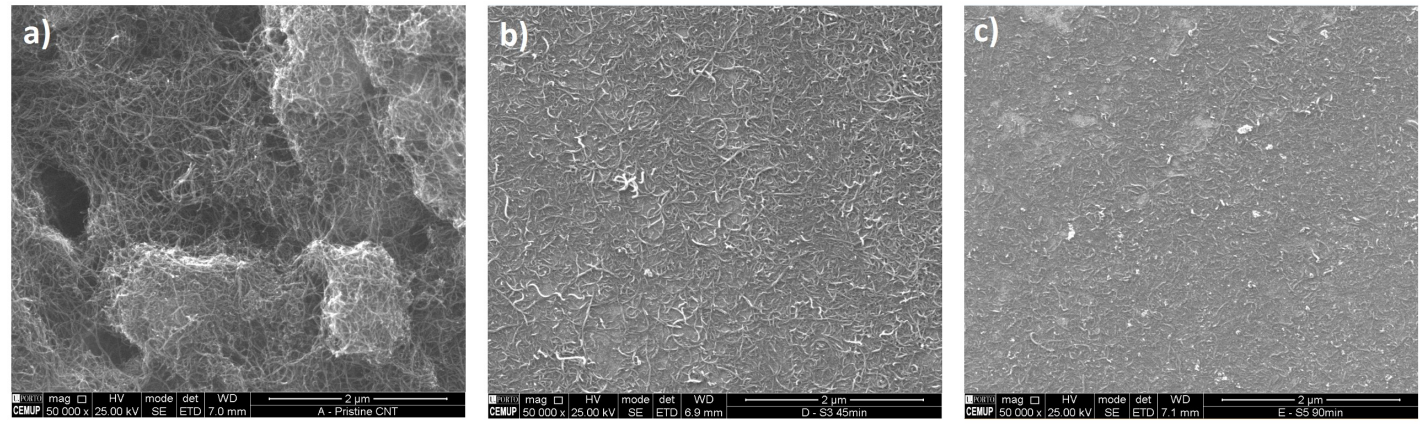

Figure 3: SEM analysis of a) pristine b) 45-minute functionalized MWCNTs and c) 90-minute functionalized MWCNTs

\subsection{Mechanical pronerties of bio-based resin nano-comnosites}

Nanocomposite samples were manufactured for the mechanical characterization containing different concentrations of pristine and 30 minutes functionalised MWCNTs. A digital image correlation system was used to detect the strain-field distribution during the experiments. The method previously described was applied for obtaining the specimens to characterise according to the ASTM D638 standard, with the aim of studying the influence of the MWCNTs type and concentration on the final mechanical properties of the nanocomposites. Neat resin samples of SR GreenPoxy ${ }^{\circledR}$ 33/SZ 8525 were also produced as benchmark case-study. In Figure 4 and Figure 5, the mechanical properties in

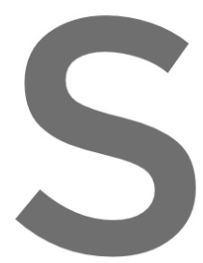
terms of tensile stress at break and Elastic modulus are respectivdly reported. Particularly the mean values of the physical pro concentrations analysed. of tensile stress at break and nanocomposites produce
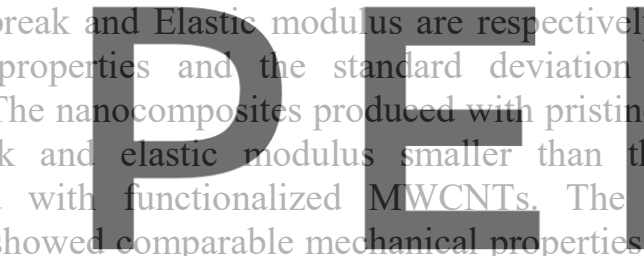
functionalized MWCNTs-s
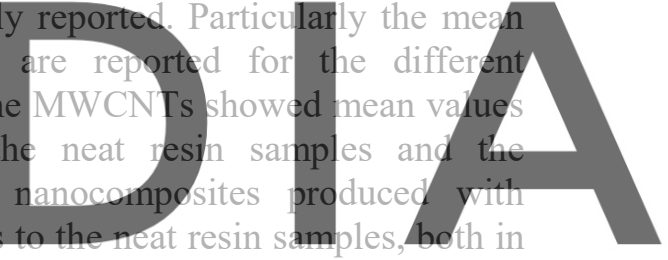

terms of elastic modulus and tensile stress at break, when a percentage of $0.25 \%$ of functionalized

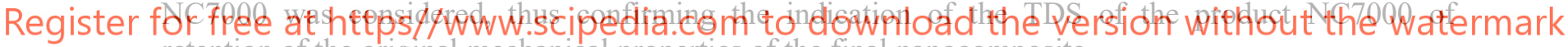
retention of the original mechanical properties of the final nanocomposite.

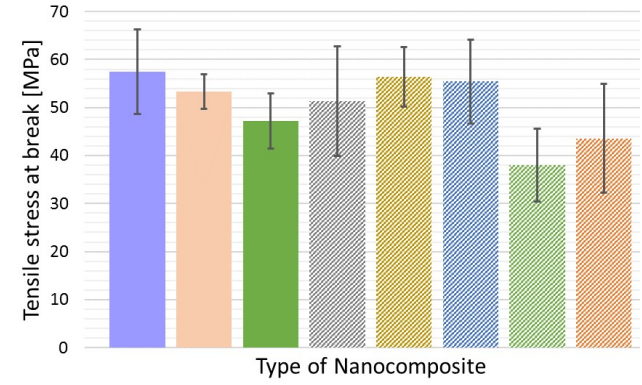

Figure 4: Mean values and standard deviation of tensile stress at break for neat resin, nanocomposites produced with pristine CNTs (MWCNTs) and with functionalized ones (fMWCNTs) at different concentrations.

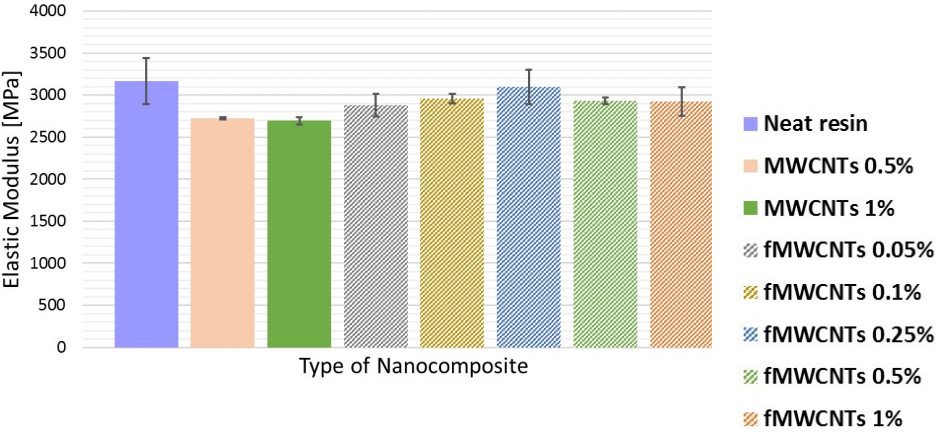

Figure 5: Mean values and standard deviation of Elastic modulus at break for neat resin, nanocomposites produced with pristine CNTs (MWCNTs) and with functionalized ones (fMWCNTs) at different concentrations. 


\section{CONCLUSIONS}

In view of nanocomposite manufacturing, chemical functionalization by carboxylic group was performed on pristine MWCNTs by means of mixture acid to improve their dispersion within a biosourced resin system. It was observed that for the functionalized MWCNTs, a change of behaviour from hydrophobic to hydrophilic led to increase the solubility of MWCNTs within an organic solvent. DMF was used to differentiate the functionalized and non-functionalized part. Amount of yield versus time of functionalization followed the first order reaction kinetics and the graph was fit by an exponential decay, showing that the amount of functionalized product decreases with the time of functionalization. From the TGA curve, it was observed that the increase in weight loss from pristine to functionalized MWCNTs can be explained by the presence of carboxylic group at the surface of MWCNTs. SEM images showed that the MWCNTs were well dispersed but length of the MWCNTs was shorter than the pristine ones. The decrease in length of MWCNTs was attributed to the acid treatment; nevertheless, the functionalized MWCNTs were not in the form of agglomerates and were well dispersed into the resin. It was found out that with an increase of synthesis time, the overall yield decreases but the percentage of functionalized product inside the yield quantity remains the same. Therefore, the time of reaction consumed more pristine MWCNTs but did not change the final functionalized MWCNTs product. The functionalized MWCNTs based nanocomposites showed mechanical tensile properties higher than the pristine-based ones produced according to the same concentration. For specific concentrations both tensile elastic modulus and tensile stress at break were comparable to the ones of the neat resin samples.

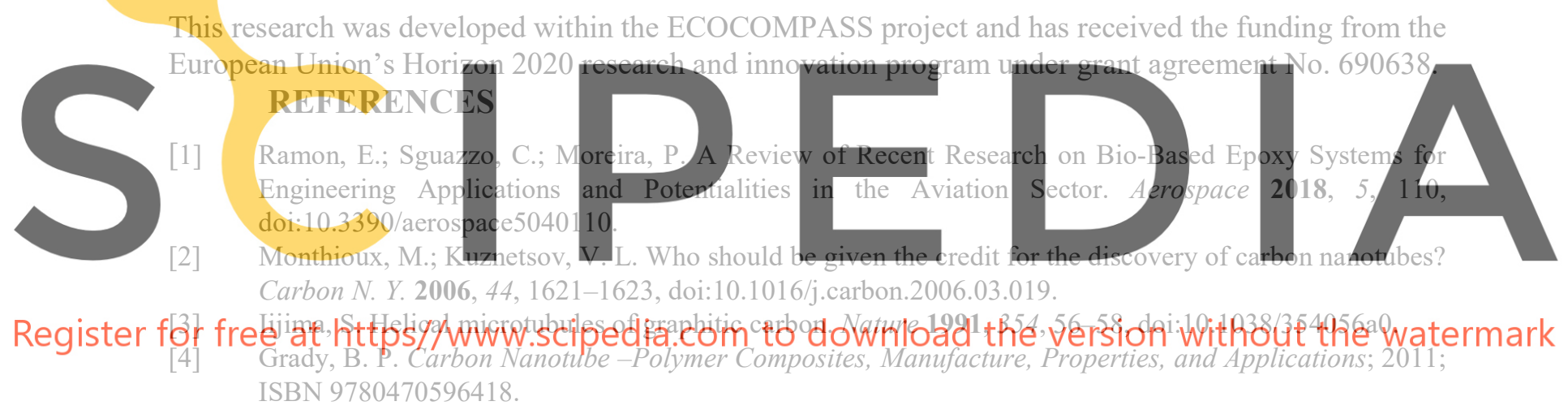

[5] Sahoo, N. G.; Rana, S.; Cho, J. W.; Li, L.; Chan, S. H. Polymer nanocomposites based on functionalized carbon nanotubes. Prog. Polym. Sci. 2010, 35, 837-867, doi:10.1016/j.progpolymsci.2010.03.002.

[6] Mahmood, N.; Islam, M.; Hameed, A.; Saeed, S.; Khan, A. N. Polyamide-6-based composites reinforced with pristine or functionalized multi-walled carbon nanotubes produced using melt extrusion technique. J. Compos. Mater. 2014, 48, 1197-1207, doi:10.1177/0021998313484779.

[7] Ma, P.-C.; Siddiqui, N. A.; Marom, G.; Kim, J.-K. Dispersion and functionalization of carbon nanotubes for polymer-based nanocomposites: A review. Compos. Part A Appl. Sci. Manuf. 2010, 41, 1345-1367, doi:10.1016/J.COMPOSITESA.2010.07.003.

[8] Nanocyl TDS - NanoCyl NC7000 Available online: https:/www.nanocyl.com/wpcontent/uploads/2016/07/DM-TI-02-TDS-NC7000-V08.pdf (accessed on Apr 1, 2019).

[9] Osorio, A. G.; Silveira, I. C. L.; Bueno, V. L.; Bergmann, C. P. H2SO4/HNO3/HCl-Functionalization and its effect on dispersion of carbon nanotubes in aqueous media. Appl. Surf. Sci. 2008, 255, 24852489, doi:10.1016/j.apsusc.2008.07.144.

[10] Men, X. H.; Zhang, Z. Z.; Song, H. J.; Wang, K.; Jiang, W. Functionalization of carbon nanotubes to improve the tribological properties of poly(furfuryl alcohol) composite coatings. Compos. Sci. Technol. 2008, 68, 1042-1049, doi:10.1016/j.compscitech.2007.07.008.

[11] Sicomin TDS - SR GreenPoxy $33 /$ SZ 8525 Available online: http://www.sicomin.com/datasheets/product-pdf1 165.pdf. 